\title{
"ABERRANT" NEURONAL STIMULATION AND "CANNABIS PSYCHOSIS" - HYPOTHESIS TO A BIOLOGICAL PLAUSIBILITY!
}

\author{
Nileswar Das \\ Department of Psychiatry and National Drug Dependence Treatment Centre, All India Institute of Medical Sciences, \\ New Delhi, India
}

received: 28.3.2021;

revised: 31.3.2021;

accepted: 18.4 .2021

\begin{abstract}
SUMMARY
Western democracies continue to 'legalize recreational cannabis use' after its 'medicinal approval', while India continues to debate whether to 'decriminalize' cannabis or not. One of the strongest arguments against cannabis decriminalization is cannabis dependence and the risk of psychosis, at-least in the vulnerable groups. Endocannabinoids are involved in neuronal proliferation and differentiation during 'patterning of the central nervous system'. Aberrant neurostimulation caused by repeated heavy exocannabinoid exposure may increase the probability of pro-psychotic experiences. Various animal and human studies have demonstrated increased but abnormal activation of cortical and subcortical areas due to chronic cannabis use. Some of these areas are involved in the pathogenesis of psychosis or schizophrenia. A review of the literature was done to hypothesize the possible role of cannabis to cause (or precipitate) psychosis through repeated "aberrant neurostimulation". The 'aberrant neurostimulation model of cannabis psychosis' may explain pathogenesis, individual vulnerabilities and developing therapeutic strategies for this debated entity. In future, well designed placebo-controlled studies may find the answer with more confidence.
\end{abstract}

Key words: cannabis - cannabinoids - substance induced - psychosis - schizophrenia - hypothesis

$* * * * *$

\section{INTRODUCTION}

Cannabis is the most widely used illegal drug in the world, with an estimated 192 million users worldwide in 2016 (United Nations Office on Drugs and Crime 2018). Recent national survey "Magnitude of Substance use in India, 2019" summarizes as cannabis being the second most commonly used substance in India, with 31 million individuals $(2.8 \%$ of the Indian population) reported to use cannabis within the previous year (Ambekar et al. 2019).

$\Delta$ 9-tetrahydrocannabinol (THC) is the primary psychoactive compound of cannabis, obtained from the plant Cannabis sativa. It is a highly lipophilic molecule that readily crosses the blood-brain barrier to act through the CB1 cannabinoid receptor which mediates its psychological and behavioural effects such as, on memory (hippocampus), on emotional responses (amygdala), on cognition (cerebral cortex), on motivation (limbic forebrain) and on motor coordination (cerebellum) (Iversen 2012, Hu \& Mackie 2015).

Psychosis is one of the most serious psychiatric illnesses with often chronic, relapsing, deteriorating courses. Therefore any avoidable risk factor can significantly add to the quality of life of an individual and help to preserve many disability-adjusted life-years (DALY) (He et al. 2020). Cannabis has long been postulated to be one of the causes of 'drug-induced psychosis', with or without complete reversibility, unlike classical substanceinduced psychiatric disorders. In recent years much attention has been provided to understand how cannabis could have been linked to the precipitation or causation of psychosis. We hereby hypothesize the 'aberrant neurostimulation hypothesis for cannabis-related psychosis' based on the review of the existing literature.

\section{CANNABIS USE CAN CAUSE ABERRANT NEUROSTIMULATION}

There is ample evidence that regular cannabis use can cause "aberrant neurostimulation". Not only the reward pathway but also stimulation of several dopaminergic and glutamatergic neurons, including the effects on the Default mode network (DMN) has been linked with chronic cannabis use (Whitfield-Gabrieli et al. 2018).

Recent animal studies have established that juvenile male rats exposed in utero to THC, manifest an imbalance in the ratio between excitatory and inhibitory inputs (E/I ratio) onto dopamine neurons in the Ventral tegmental area (VTA), which contributes to the enhanced excitability of those neurons (Frau et al. 2019). Prenatal THC exposure also alters the expression of CB1, dopamine, and glutamate receptor genes, resulting in impaired striatal synaptic plasticity and enhanced drug-seeking behaviours in adult rat offspring (Spano et al. 2007; Tortoriello et al. 2014).

In human studies, administration of THC has been associated with subjective and cognitive alterations (via increased striatal glutamate and dopamine concentration) and loss of functional connectivity (FC) between Nucleus accumbens (NAc) and cortical areas. An increase in glutamate concentrations and alteration in cognitive function was dose (of THC) dependent (Mason et al. 2019).

A recent fMRI based meta-analysis has shown that, during the phase of reward anticipation, individuals with cannabis dependence have decreased striatal activation as compared to the healthy controls. While during reward outcome, individuals with cannabis dependence have higher activation of ventral striatum - suggesting altered reward 
processing (Luijten et al. 2017). Cannabis use group as compared to healthy controls has also shown decreased functional connectivity between caudal anterior cingulate cortex (ACC) and dorsolateral prefrontal cortex/ orbitofrontal cortex (DLPFC/ OFC) (Camchong et al. 2017).

Preclinical studies in rodents have suggested that gamma oscillations arising from GABA receptors that connect cortical networks are important for cognitive function (Wang \& Buzsáki 1996). CB1 receptor activetion by THC agonists may disrupt gamma oscillations through inhibitory interneurons, suggesting that gamma oscillations may be disrupted in cannabis users (Hájos et al. 2000, Skosnik et al. 2012). A recent electrophysiological study has found that individuals with cannabis use disorder (CUD) have decreased delta and increased theta, beta, and gamma powers compared to controls, suggesting increased cortical activation in resting state and disinhibition of inhibitory functions that may interrupt cognitive processes. Increased interhemispheric and intrahemispheric electrical activity is also found in CUD. This increased cortical activity reflects a "noisy brain" in individuals with CUD (Prashad et al. 2018).

In summary, various neurochemical, neuroimaging and neurophysiological studies have demonstrated increased but abnormal activation of various cortical and subcortical areas (often these areas are also linked to psychosis or schizophrenia; e.g. dopamine and glutamate pathways) as a result of chronic cannabis use.

\section{CANNABIS AND PSYCHOSIS: ABERRANT NEUROSTIMULATION AS A MODEL}

Kapur's (2003) "aberrant salience" is one of the most accepted models explaining the genesis of abnormal behavioural responses associated with psychosis (Apopheny) (Kapur 2003). Animal models of aberrant motivational salience result because of out-of-context signalling of dopamine in the ventral striatum, which may, in turn, be driven by abnormal regulation of subcortical dopamine transmission by the PFC and hippocampus (Roiser et al. 2013). Similar associations of defective neuronal signalling and (positive and/or negative) psychotic experiences have also been found in recent human studies. Even in individuals without clinical psychosis, studies have found a strong positive correlation between aberrant salience score (measured by Aberrant Salience Inventory) and cannabis use (amount and frequency) (Bernardini et al. 2018, Chun et al. 2019).

Endocannabinoids are known to be involved in neuronal proliferation, migration, differentiation and survival during 'patterning of the central nervous system'. Aberrant neurostimulation caused by repeated heavy cannabis use (exo-cannabinoids), especially in a developing (adolescent) brain increases the probability of pro-psychotic experiences (Di Forti et al. 2019).

Adolescent onset (before age 15 years) CUD has been associated with activation differences in the frontoparietal (increased) and visual association (decreased) regions and has shown poorer executive planning scores compared to non-initiators (Tervo-Clemmens et al. 2018). The endocannabinoid (eCB) system is involved in extensive re-organisation of grey matter and white matter during adolescent brain development. Therefore, CUD in youth is associated with memory loss, hippocampal volume loss and left parietal lobe dysfunction especially in those with early-onset schizophrenia-spectrum disorders (EOSS). Similarly, a higher reduction in functional anisotropy of left inferior longitudinal fasciculus (ILF) was found in youth with CUD, which is also implicated in the pathogenesis of EOSS (James et al. 2013, Epstein \& Kumra 2015).

In a before-after (cannabis use) fMRI study, Whitfield-Gabrieli and colleagues found that, at baseline, relative to controls, patients (psychosis with CUD) exhibited DMN hyperconnectivity that correlated with positive psychotic symptom severity, and reduced anticorrelation between the DMN and the executive control network (ECN). Cannabinoid administration (3.6\% THC cannabis cigarette smoked or a $15 \mathrm{mg} \Delta 9$-THC pill ingested) reduced DMN hyperconnectivity and increased DMN-ECN anticorrelation (Whitfield-Gabrieli et al. 2018).

\section{IMPLICATIONS}

The plastic nature of several functional pathways including DMN in response to (endogenous and exogenous) cannabinoids, may have both pathogenic and therapeutic implications. Aberrant neuroplastic changes with heavy cannabis smoking may explain the pathways involved in complex mental illnesses like psychosis. At the same time may provide a window of opportunity to study the potential role of cannabinoids as pharmacotherapeutic agents for the treatment of psychotic illness in the near future (Whitfield-Gabrieli et al. 2018, Davies and Bhattacharyya 2019, Sarris et al. 2020).

Certain genetic polymorphisms (e.g. COMT-Val158Met functional polymorphism in the catecholamine metabolising enzyme catechol-o-methyltransferase gene) may add to the increased risk of development of psychotic phenomena (e.g. delusion-like experiences) associated with adolescent-onset heavy cannabis use (Henquet et al. 2009). This may explain why certain populations are more vulnerable to develop psychosis after aberrant stimulation due to chronic heavy cannabis use, than others.

\section{CONCLUSION}

Current literature is inadequate to solve the neverending saga between cannabis and psychosis. Despite the recent change in the legal status of cannabis, a further search should continue to explore the plausibility of cannabis associated (induced or precipitated) psychosis as a clinical entity. One of the limitations of the proposed 'aberrant neurostimulation' model is the lack of emphasis on psychological, social and phenomenological aspects of cannabis psychosis, so does the available literature. However, the literature does hint towards the abnormal 
neurostimulation associated with cannabis use and as a plausible mechanism, explaining how early-onset heavy cannabis use may lead to psychosis. Further studies do require to establish or refute the proposed hypothesis.

\section{Acknowledgements:}

Dr Siddharth Sarkar, Associate professor of Psychiatry, Department of Psychiatry and National Drug Dependence Treatment Centre, All India Institute of Medical Sciences, New Delhi, India, 110029.

\section{Conflict of interest: None to declare.}

\section{References}

1. Ambekar A, Agrawal A, Rao $R$ et al.: on behalf of the group of investigators for the National Survey on Extent and Pattern of Substance Use in India (2019). Magnitude of Substance Use in India. New Delhi: Ministry of Social Justice and Empowerment, Government of India, 2019

2. Bernardini F, Gobbicchi C, Attademo L et al.: Cannabis Use, Psychotic Like Experiences and Aberrant Salience in a Sample of Belgian Students. J Nerv Ment Dis 2018; 206:493-500

3. Camchong J, Lim KO, Kumra S. Adverse Effects of Cannabis on Adolescent Brain Development: A Longitudinal Study. Cereb Cortex 2017; 27:1922-1930

4. Chun CA, Brugger P, Kwapil TR. Aberrant Salience Across Levels of Processing in Positive and Negative Schizotypy. Front Psychol 2019; 10:2073

5. Davies C, Bhattacharyya S. Cannabidiol as a potential treatment for psychosis. Ther Adv Psychopharmacol 2019; 9: 2045125319881916

6. Di Forti M, Quattrone D, Freeman TP et al.: The contribution of cannabis use to variation in the incidence of psychotic disorder across Europe (EU-GEI): a multicentre case-control study. Lancet Psychiatry 2019; 6: 427-436.

7. Epstein KA, Kumra S. White matter fractional anisotropy over two time points in early onset schizophrenia and adolescent cannabis use disorder: A naturalistic diffusion tensor imaging study. Psychiatry Res 2015; 232: 34-41.

8. Frau R, Miczán V, Traccis F et al. Prenatal THC exposure produces a hyperdopaminergic phenotype rescued by pregnenolone. Nat Neurosci 2019; 22: 1975-1985.

9. Hájos N, Katona I, Naiem SS et al. Cannabinoids inhibit hippocampal GABAergic transmission and network oscillations. Eur J Neurosci 2000; 12: 3239-3249.

10. He H, Liu Q, Li $N$ et al. Trends in the incidence and $D A L Y$ s of schizophrenia at the global, regional and national levels: results from the Global Burden of Disease Study 2017. Epidemiol Psychiatr Sci 2020; 29: e91.

11. Henquet C, Rosa A, Delespaul P et al.: COMT ValMet moderation of cannabis-induced psychosis: a momentary assessment study of "switching on" hallucinations in the flow of daily life. Acta Psychiatr Scand 2009; 119:156-160
12. Hu SS-J, Mackie K: Distribution of the Endocannabinoid System in the Central Nervous System. Handb Exp Pharmacol 2015; 231: 59-93

13. Iversen L: How cannabis works in the human brain. Marijuana and madness. Cambridge University Press, Cambridge 2012; 1-11

14. James A, James C, Thwaites T. The brain effects of cannabis in healthy adolescents and in adolescents with schizophrenia: a systematic review. Psychiatry Res 2013; 214: 181-189.

15. Kapur S. Psychosis as a state of aberrant salience: a framework linking biology, phenomenology, and pharmacology in schizophrenia. Am J Psychiatry 2003; 160:13-23

16. Luijten $M$, Schellekens AF, Kühn $S$ et al.: Disruption of Reward Processing in Addiction: An Image-Based Metaanalysis of Functional Magnetic Resonance Imaging Studies. JAMA Psychiatry 2017; 74:387-398

17. Mason NL, Theunissen EL, Hutten NRPW et al. Cannabis induced increase in striatal glutamate associated with loss of functional corticostriatal connectivity. Eur Neuropsychopharmacol 2019; 29: 247-256

18. Prashad S, Dedrick ES, Filbey FM. Cannabis users exhibit increased cortical activation during resting state compared to non-users. Neuroimage 2018; 179: 176-186

19. Roiser JP, Howes OD, Chaddock CA et al. Neural and Behavioral Correlates of Aberrant Salience in Individuals at Risk for Psychosis. Schizophr Bull 2013; 39:1328-1336

20. Sarris J, Sinclair J, Karamacoska D et al. Medicinal cannabis for psychiatric disorders: a clinically-focused systematic review. BMC Psychiatry 2020; 20:24

21. Skosnik PD, D'Souza DC, Steinmetz AB et al. The effect of chronic cannabinoids on broadband EEG neural oscillations in humans. Neuropsychopharmacology 2012; 37: 2184-2193

22. Spano MS, Ellgren M, Wang $X$ et al. Prenatal cannabis exposure increases heroin seeking with allostatic changes in limbic enkephalin systems in adulthood. Biol Psychiatry 2007; 61:554-563

23. Tervo-Clemmens B, Simmonds D, Calabro FJ et al. Early Cannabis Use and Neurocognitive Risk: A Prospective Functional Neuroimaging Study. Biol Psychiatry Cogn Neurosci Neuroimaging 2018; 3:713-725

24. Tortoriello G, Morris CV, Alpar A et al. Miswiring the brain: 49-tetrahydrocannabinol disrupts cortical development by inducing an SCG10/stathmin-2 degradation pathway. EMBO J 2014; 33:668-685

25. United Nations Office on Drugs and Crime. World Drug Report 2018. United Nations publication, Sales No. E. 18. XI. 9 2018. UNODC 2018

26. Wang XJ, Buzsáki G. Gamma oscillation by synaptic inhibition in a hippocampal interneuronal network model. J Neurosci 1996; 16:6402-6413

27. Whitfield-Gabrieli S, Fischer AS, Henricks AM et al. Understanding marijuana's effects on functional connectivity of the default mode network in patients with schizophrenia and co-occurring cannabis use disorder: A pilot investigation. Schizophr Res 2018; 194:70-77

\section{Correspondence:}

Nileswar Das, MD

Department of Psychiatry and National Drug Dependence Treatment Centre,

All India Institute of Medical Sciences

First floor, CMET, Ansari Nagar, New Delhi, 110029, India

E-mail:dr.nileswar@gmail.com 